\title{
Tumor neuroendocrino primario testicular: reporte de un caso y revisión de la literatura
}

\author{
Primary neuroendocrine tumor of the testis. A case report and literature review \\ Francisco Ortega ${ }^{1,2}$, Nelson Urdaneta ${ }^{1}$, Nicolás Yañez ${ }^{1,2}$, Erik Morales ${ }^{3,4}$, Valeria Monasterio ${ }^{3,4}$, Dahiana Pulgarr, ${ }^{1,2}$ René Henriquez ${ }^{5}$
}

\section{Resumen}

Los tumores neuroendocrinos primarios del testículo son una entidad muy infrecuente, dando cuenta del $1 \%$ de las neoplasias testiculares. Clínicamente se presentan indolentes, por una masa testicular. En tumores localizados, rara vez presentan síndrome carcinoide. La orquiectomía radical es el tratamiento de elección; el pronóstico es excelente en etapas precoces. Las terapias adyuvantes no han mostrado utilidad.

Se presenta un caso de un hombre de 53 años con una masa testicular palpable de larga evolución. Se realiza orquiectomía radical. La biopsia muestra un tumor neuroendocrino bien diferenciado. Sin evidencia de metástasis en etapificación. Se define en comité oncológico, realizar seguimiento.

Palabras clave: neuroendocrino; carcinoide; neoplasia; tumor; testículo.

\begin{abstract}
The primary neuroendocrine tumors of the testis are an infrequent entity, accounting for less than $1 \%$ of all testicular neoplasms. Clinically they are presented by a testicular mass of indolent curse. In localized tumors, they rarely have carcinoid syndrome. Radical orchiectomy is the treatment of choice, and the prognosis is excellent in the early stages. Adjuvant therapies have not shown utility.

We present a case of a 53-year-old man with a long-standing testicular mass. He received radical orchiectomy. The pathology report shows a well-differentiated neuroendocrine tumor with no evidence of metastasis in staging. Tumor board defined follow-up.
\end{abstract}

Keywords: neuroendocrine; carcinoid; neoplasm; tumor; testis

Fecha de envío: 23 de julio de 2019 - Fecha de aceptación: 9 de diciembre de 2019

\section{Introducción}

Los tumores neuroendocrinos (TNE) son tumores derivados del sistema neuroendocrino difuso, el cual se encuentra compuesto de células productoras de péptidos y aminas, pudiendo producir diferentes hormonas dependiendo del sitio de origen (DeVita, 2011). Comúnmente derivan del tejido gastrointestinal o pulmonar. Datos del programa SEER (Surveillance, Epidemiology, and End Results) del $\mathrm{NCl}$ (National Cancer Institute Database) de EEUU, muestran que la ubicación gastrointestinal representa un $55 \%$ y la broncopulmonar un 30\% (Maggard et al., 2004). A su vez los tumores neuroendocrinos primarios o carcinoide del testículo son muy infrecuentes, dando cuenta de menos del $1 \%$ de todas las neoplasias testiculares y un 0,2\% de las neoplasias neuroendocrinas (Berdjis \& Mostofi, 1977; Kato et al., 2003)

\section{Caso clínico}

Paciente masculino de 53 años, sin antecedentes mórbidos. Refiere tumor testicular izquierdo, desde los 18 años con crecimiento progresivo en últimos 4 meses. No presenta diarrea, bochornos $u$ otros síntomas. Se realiza ecografía testicular en mayo 2018 que muestra tumor en testículo izquierdo 6,0 ×5,3 x 3,8 cms. Marcadores tumorales negativos pre operatorios (AFP: 3, $1 \mathrm{ng} / \mathrm{mL}$; BHCG: 0,10

(1) Servicio de Oncología Hospital Regional de Talca, Chile.

(2) Facultad de Medicina, Universidad Católica del Maule, Chile.

(3) Unidad de Anatomía Patológica Hospital Regional de Talca, Chile.

(4) Departamento de Ciencias Preclínicas, Facultad de Medicina, Universidad Católica del Maule, Chile

(5) Servicio de Urología, Hospital Regional de Talca, Chile.

Autor de correspondencia: fortega.gonzalez@gmail.com 
$\mathrm{mUl} / \mathrm{mL}$ ). En junio 2018 se realiza orquiectomía ampliada izquierda. Biopsia muestra tumor de $5 \mathrm{cms}$. con proliferación neoplásica neuroendocrina de patrón sólido, de células poligonales con escasa atipia y ausencia de figuras mitóticas. Sin necrosis. Indice mitotico $<2 / 2 \mathrm{~mm} 2$. Con presencia de invasión linfovascular, compromiso parcial de epidídimo. Sin compromiso albuginea ni túnica vaginal. Rete testis y cordón espermático libre de tumor. (Figura 1). Hallazgos compatibles con tumor neuroendocrino bien diferenciado. Estudio inmunohistoquímico muestra: Ki67 de 20\%.CD117 negativo. Citokeratinas totales: positiva en células tumorales con patrón de membrana. CK7: negativo. CK20: negativo. PSA: negativo. CD56: positiva intensa en células tumorales. Sinaptofisina: positiva intensa en células tumorales. Cromogranina: positiva en células tumorales. (Figura 2 a 4)

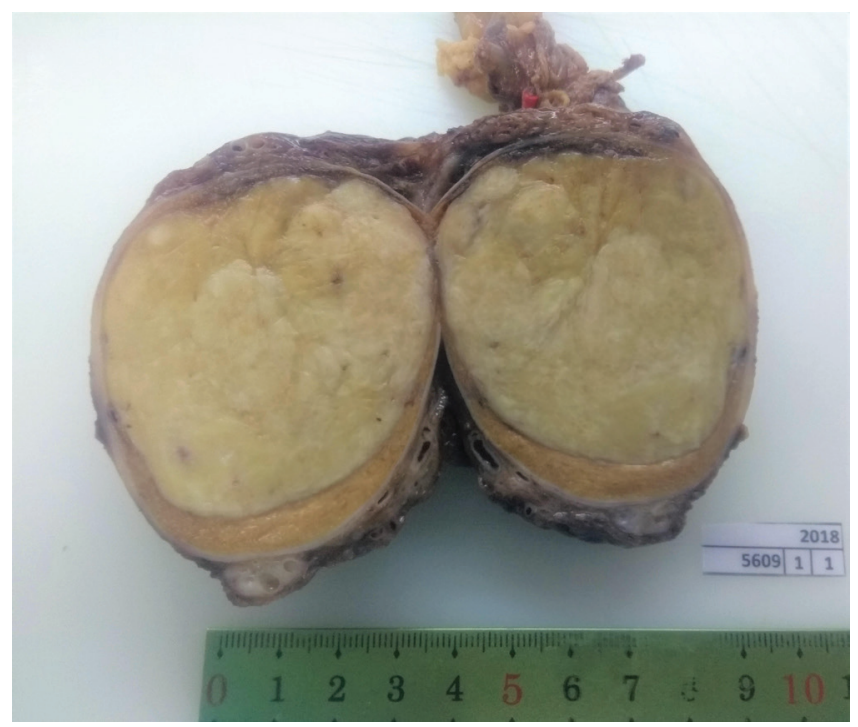

Figura 1: Imagen macroscópica que muestra extensa masa tumoral testicular bien delimitada, compresiva, no encapsulada, homogénea, sin necrosis ni hemorragia.

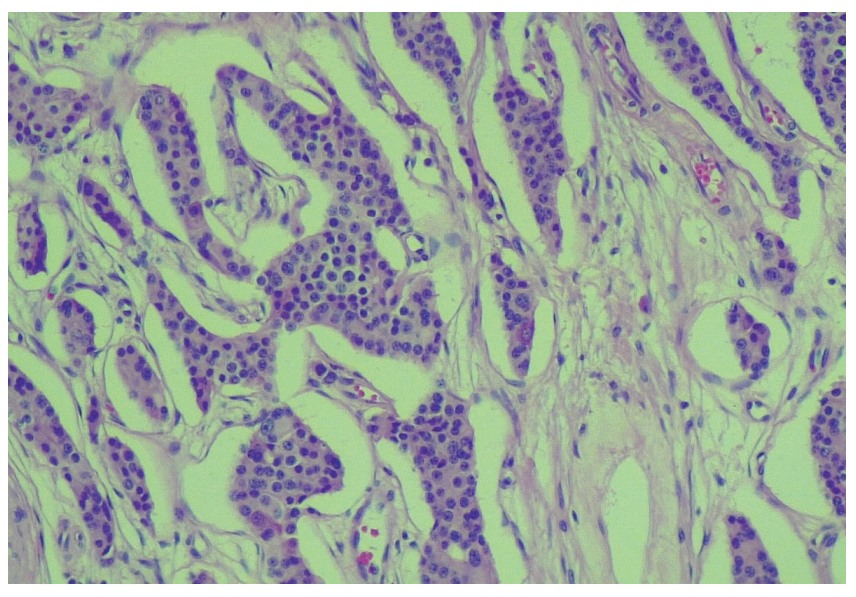

Figura 2: Microfotografía (H\&E, 100X) que muestra en mitad derecha proliferación neoplásica neuroendocrina de patrón en cordones y nidos, y en mitad izquierda presencia de túbulos seminíferos (Sin evidencia de neoplasia germinal in situ).

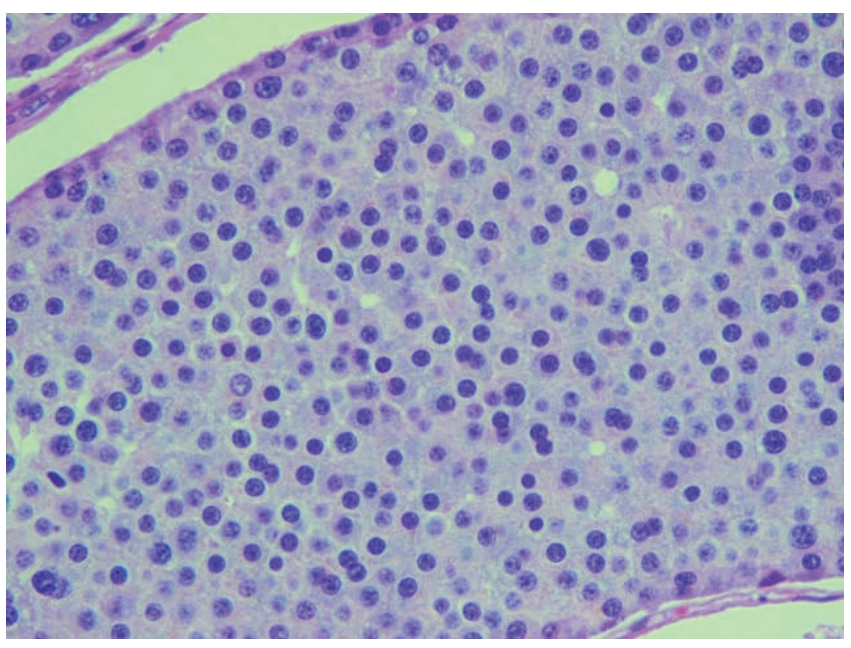

Figura 3: Microfotografía (H\&E, 400X) que muestra detalle de la proliferación neoplásica neuroendocrina, de patrón sólido, de células poligonales con escasa atipia y ausencia de figuras mitóticas.

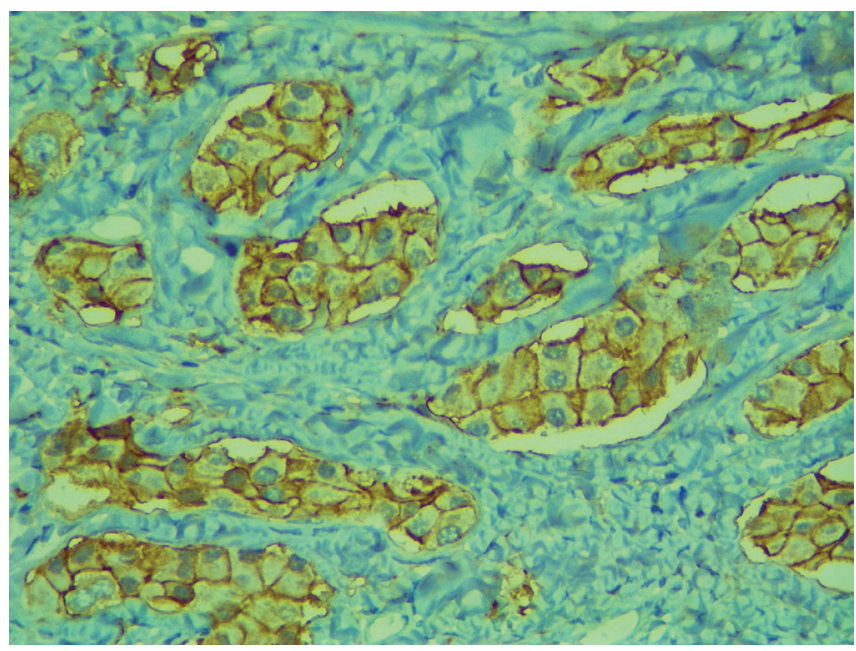

Figura 4: Microfotografía de tinción de inmunohistoquímica (CD56, 400X) que muestra intensa positividad de la proliferación neoplásica.

Tomografía computada (TC) de tórax, abdomen y pelvis en julio 2018 no muestra signos de diseminación ni presencia de otros tumores.

Evaluado en comité de uro-oncología, se decidió seguimiento clínico e imagenológico siguiendo pautas habituales de cáncer testicular localizado. Al momento de la publicación no hay evidencias de metástasis durante el seguimiento.

\section{Discusión}

Obendorfer \& Frankfurt (1907) acuñaron el término carcinoide para separar un grupo de tumores del intestino delgado que se comportaban menos agresivos que los carcinomas convencionales. Años después, Gosset \& Masson (1914) sugieren que estos tumores derivan de las células de Kulchitsky demostrando 
gránulos argentafines en dichas células. Williams \& Sandler (1963) posteriormente presentan su clasificación basada en la división embriológica del intestino, dividiéndolo en proximal, medio y distal. Actualmente, la organización mundial de la salud, clasifica estos tumores como neuroendocrinos (Bosman et al., 2010), siendo el término carcinoide aplicado para tumores neuroendocrinos bien diferenciados originados en el tracto digestivo, broncopulmonar, o sitios infrecuentes, como el testículo.

Los TNE testiculares son especialmente infrecuentes. Clínicamente se presentan de forma indolente, como aumento de volumen testicular o una masa pequeña (Zavala-Pompa et al., 1993). En general, el tamaño tumoral determina la probabilidad de desarrollar un síndrome carcinoide. Se reporta que el 50\% de los tumores metastásicos tiene un síndrome carcinoide, mientras un 5,6\% de los tumores sin metástasis tienen este síndrome. (Park et al., 2006) Los tumores neuroendocrinos testiculares pueden ser encontrados en tres escenarios clínicos: (i) como componente de un teratoma; (ii) como una lesión metastásica y (iii) como una neoplasia neuroendocrina de novo (primaria). Los primeros 2 escenarios son relativamente fáciles de explicar, sin embargo el tercer escenario representa un reto, pues la presencia de células neuroendocrinas (o células de Kulchitsky) no han sido descritas en el testículo. Una explicación propuesta, es la suposición de que existen restos de células neuroendocrinas de la etapa embrionaria, que a su debido tiempo son capaces de formar una masa tumoral (Reyes et al., 2003).

Un 10\% de los tumores neuroendocrinos del testículo son metástasis de otro primario. Es por esto que es necesario un estudio complementario con enfoque multimodal. Como la mayoría de los TNE se originan en el tracto gastrointestinal, según el grado de sospecha, se recomienda estudio contrastado con bario, o eventualmente cápsula endoscópica. Además, se recomienda TC contrastada para detección de metástasis hepáticas y extensión mesentérica (Neely \& Gray, 2011; Hung-Keng et al., 2014). Estudios de medicina nuclear como el cintigrama de receptores de somatostatina o PET 68Ga-DOTATATE, presentan mayor sensibilidad para la detección de tumor primario y metástasis, pudiendo ser utilizados de forma complementaria. (Ramage et al., 2005; Al-Nahhas et al., 2007)

La orquiectomía radical es el tratamiento de elección para el TNE testicular. Terapias adyuvantes como quimioterapia y radioterapia han mostrado ser de escasa utilidad (Stroosma \& Delaere 2008).

El pronóstico del TNE testicular depende del tamaño tumoral, la asociación con teratoma, y la presencia de metástasis. Zavala-Pompa et al., (1993), muestra que tumores de mayor tamaño (7,3 vs 2,9 cms) y la presencia de síndrome carcinoide son factores de mal pronóstico, aumentando la posibilidad de metástasis. En el caso de tumores localizados, el pronóstico es excelente. Wang et al., (2010) reporta 20 de 29 casos con tumor carcinoide primario testicular, todos vivos sin recurrencia a los 52 meses de seguimiento.

\section{Conclusión}

Los TNE testiculares primarios son infrecuentes y se presentan de forma indolente. Por el riesgo de ser una presentación metastásica de otro primario, debe realizarse estudio con enfoque multimodal. El tratamiento consiste en cirugía, siendo las terapias adyuvantes de poca utilidad. El pronóstico depende de factores como el tamaño y la etapa, siendo excelente en tumores localizados.

\section{Fuentes de financiamiento}

El trabajo no presenta fuentes de financiamiento

\section{Contribuciones y conflictos declarados por los autores}

Los autores no reportan conflictos de interés.

\section{Referencias}

Al-Nahhas A, Win Z, Szyszko T, Singh A, Khan S \& Rubello D. (2007). What can gallium-68 PET add to receptor and molecular imaging? European journal of nuclear medicine and molecular imaging 34, 1897-1901.

Berdjis C \& Mostofi F. (1977). Carcinoid tumors of the testis. The Journal of Urology 118, 777-782.

Bosman F, Carneiro F, Hruban R \& Theise N. (2010). Tumours of the stomach. In: WHO Classification of Tumours of the Digestive System. 4th Edition, pp. 37-66. Lyon: International Agency for Research on Cancer

DeVita, Vincent T. (2011), Jr. Carcinoid Tumors and Carcinoid Syndrome. In: DeVita, Cancer: Principles and Practice of Oncology, 10th Edition, pp. 8518 - 8568. Philadelfia: Lippincott Williams \&Wilkins.

Gosset A \& Masson P. (1914). Tumeurs endocrines de l'appendice. Presse Medicine 22, 237-240.

Hung-Keng L, Yi-Hsiu Huang E, Lin A \& Kuang-Kuo C. (2014), Testicular carcinoid tumor: A case report and literature review, Urological Science 25, 143-145

Kato N, Motoyama T, Kameda N, Hiruta N, Emura I, Hasegawa G, Murata T, Kimura M, Tsuda H \& Ishihara T. (2003). Primary carcinoid tumor of the testis: immunohistochemical, ultrastructural and FISH analysis with review of the literature. Pathology International 53, 680-685. 
Ortega et al.

Maggard MA, O'Connell JB \& Ko CY. (2004) Updated population-based review of carcinoid tumors. Annals of Surgery 240, 117-122

Neely D \& Gray S. (2011). Primary carcinoid tumour of the testis. The Ulster medical journal 80, 79-81

Obendorfer S \& Frankfurt Z. (1907). Karzinoide tumoren des duenndarms. Pathology 1, 426-430

Park SB, Kim JK \& Cho KS. (2006). Imaging findings of a primary bilateral testicular carcinoid tumor associated with carcinoid syndrome. Journal of Ultrasound Medicine 25, 413-416.

Ramage JK, Davies AH, Ardill J, Bax N, Caplin M, Grossman A, Hawkins R, McNicol AM, Reed N, Sutton R, Thakker R, Aylwin S, Breen D, Britton K, Buchanan K, Corrie P, Gillams A, Lewington V, McCance D, Meeran K \& Watkinson A. (2005). Guidelines for the management of gastroenteropancreatic neuroendocrine (including carcinoid) tumours. Gut 54(suppl 4), 1-16.
Reyes A, Moran C, Suster S, Michal M \& Dominguez H. (2003). Neuroendocrine Carcinomas (Carcinoid Tumor) of the Testis A Clinicopathologic and Immunohistochemical Study of Ten Cases. American journal of clinical pathology 120, 182-187

Stroosma OB \& Delaere K. (2008). Carcinoid tumours of the testis. BJU International 101: 1101-1105.

Williams, E.D. \& Sandler, M. (1963) The classification of carcinoid tumours. Lancet 281, 238-239.

Wang W, Guo C, Berney D, Ulbright T, Hansel D, Shen R, Ali T \& Epstein J. (2010). Primary carcinoid tumors of the testis: a clinicopathologic study of 29 cases. The American journal of surgical pathology 34, 519-524.

Zavala-Pompa A, Ro J, El-Naggar A, Ordoñez N, Amin M, Pierce $P \&$ Ayala AG. (1993). Primary carcinoid tumor of testis. Immunohistochemical, ultrastructural, and DNA flow cytometric study of three cases with a review of the literature. Cancer 72, 1726-1732. 